\title{
GROWTH OF THE EDIBLE MICROALGA ARTHROSPIRA PLATENSIS IN RELATION TO BORON SUPPLY
}

\author{
$\operatorname{AUTHOR}(\mathrm{S})$ : \\ Tadama, Shotaro; Shiraishi, Hideaki
}

\section{CITATION:}

Tadama, Shotaro ... [et al]. GROWTH OF THE EDIBLE MICROALGA ARTHROSPIRA

PLATENSIS IN RELATION TO BORON SUPPLY. International Journal of GEOMATE 2017, 12(30): 90-95

ISSUE DATE:

2017-02

URL:

http://hdl.handle.net/2433/230368

\section{RIGHT:}

Copyright (c) International Journal of GEOMATE. All rights reserved, including the making of copies unless permission is obtained from the copyright proprietors. 


\title{
GROWTH OF THE EDIBLE MICROALGA ARTHROSPIRA PLATENSIS IN RELATION TO BORON SUPPLY
}

\author{
Shotaro Tadama ${ }^{1}$ and $*$ Hideaki Shiraishi ${ }^{1}$ \\ ${ }^{1}$ Graduate School of Biostudies, Kyoto University, Japan \\ *Corresponding Author, Received: 31 May 2016, Revised: 29 Nov. 2016, Accepted: 19 Dec, 2016
}

\begin{abstract}
Arthrospira (Spirulina) platensis is an edible cyanobacterium that has been consumed worldwide as a nutrient source under the name spirulina. When preparing synthetic media for this microalga, boron is usually added to them. However, whereas boron is necessary for the $\mathrm{N}_{2}$-fixation-dependent growth of heterocystous cyanobacteria, boron requirement by A. platensis, which is non-heterocystous, has not yet been carefully examined. To examine the effect of boron on A. platensis, we prepared a boron-depleted medium in which borate concentration was below a detection limit $(0.2 \mu \mathrm{M})$, as determined by the spectrophotometric quantitation with H-resorcinol. Using this boron-depleted medium, A. platensis NIES-39 was analyzed for changes in growth, dry biomass weight and the protein, chlorophyll $a$ and C-phycocyanin contents. Experimental results showed that removal of boron from the growth medium had no detectable effect on them. A control experiment with a heterocystous cyanobacterium Anabaena sp. UTEX 2576 showed that the growth of this cyanobacterium was suppressed in a boron-depleted medium. These experiments demonstrated that boron was not required for the growth of A. platensis. Our results indicate that boron supply is not necessary for the propagation of $A$. platensis even in the regions where boron deficiency in the soil deteriorates the growth of common crops. Use of boron-free media would also be helpful to improve the reproducibility of sensitive physiological experiments such as metabolome analysis, because precipitation of the insoluble salt manganese borate, which may reduce the concentration of $\mathrm{Mn}^{2+}$ in the medium, does not occur in boron-free media.
\end{abstract}

Keywords: Cyanobacteria, Spirulina, Arthrospira platensis, Boron, H-resorcinol

\section{INTRODUCTION}

Arthrospira platensis is an edible alkalophilic cyanobacterium that has been consumed worldwide as a nutrient source and food additives [1][2]. Phycobiliproteins from this cyanobacterium are also widely used as natural colorants for foods and cosmetics [2]. Products of A. platensis and a closely related species $A$. maxima are usually marketed under the name spirulina, because they had formerly been classified in the genus Spirulina

In the artificial propagation of $A$. platensis, boric acid is usually added to its growth medium [3], as it is well known that boron is required for the growth of many photosynthetic organisms. For example, higher plants require boron for the formation and maintenance of cell wall structures [4]. Therefore, deficiency of boron in the soil causes the development of many symptoms in higher plants [4][5]. Boron is also required for the maintenance of the integrity of heterocyst cells that perform $\mathrm{N}_{2}$ fixation in heterocystous filamentous cyanobacteria [6][7]. Boron is involved in stabilizing the heterocyst envelope in these cyanobacteria [8]. However, in contrast to higher plants and heterocystous cyanobacteria, many nonheterocystous cyanobacteria are tolerant to boron deficiency [7][9].
Since A. platensis is also non-heterocystous, it is possible that boron is not necessary for the growth of this cyanobacterium. However, boron requirement by $A$. platensis has not yet been carefully examined. Knowledge about the boron requirement would be helpful for the agriculture of A. platensis, especially in the region where boron deficiency in the soil deteriorate healthy growth of many common crops [5].

Knowledge on the boron requirement by $A$. platensis is also helpful for basic studies of this cyanobacterium. In laboratory-scale experiments, inclusion of boric acid in the growth medium results in the gradual precipitation of the insoluble salt manganese borate [10], possibly causing the change in the $\mathrm{Mn}^{2+}$ concentration and gradual depletion of $\mathrm{Mn}^{2+}$ in the growth medium. This may reduce the reproducibility of sensitive physiological experiments, e.g., metabolome analysis, because manganese is an essential component of the oxygen-evolving complex in photosystem II [11], as well as many cellular enzymes (e.g., xylose isomerase, glutamine synthetase and phosphoglycerate mutase [12]), and therefore alterations in the manganese concentration may affect cellular physiology. If boron is determined to be dispensable for the growth of A. platensis, boric acid can be omitted 
from the growth medium to improve the reproducibility of sensitive physiological experiments.

In this study, we prepared a boron-depleted medium, and effects of boron on the growth and some biochemical properties of A. platensis were examined to determine whether boron depletion had any effect on this cyanobacterium.

\section{MATERIALS AND METHODS}

\subsection{Reagents}

H-resorcinol, or 1-(2,4-Dihydroxy-1-phenylazo)-8-hydroxynaphthalene-3,6-disulphonic acid disodium salt, was purchased from Tokyo Chemical Industry Co. (Tokyo). Other chemicals were purchased from Nakalai Tesque (Kyoto) and Wako Pure Chemical Industries (Osaka). All chemicals were reagent grade or better. Water was purified with the Milli-Q Advantage A10 Ultrapure Water Purification System (Merck Millipore, Darmstadt) before use. Polypropylene plasticware was used to prepare reagent solutions, to avoid boron contamination from glassware [13]

\subsection{Cyanobacterial strains}

A. platensis NIES-39 was obtained from the Microbial Culture Collection at the National Institute for Environmental Studies, Tsukuba (MCC-NIES). Anabaena sp. UTEX 2576 was obtained from the Culture Collection of Algae at the University of Texas at Austin (UTEX).

\subsection{Growth media and growth conditions}

SOT medium and boron-depleted SOT medium for $A$. platensis were prepared as described [14][15], except that the macroelement solution used to prepare the latter medium did not contain boric acid. Anabaena sp. UTEX 2576 was cultured either in $\mathrm{BG} 11_{0}$ medium [16] or in the boron-depleted $\mathrm{BG} 11_{0}$ medium that contained no added boric acid. Cyanobacterial cells were cultured in $250-\mathrm{mL}$ polycarbonate Erlenmeyer flasks (Thermo Scientific Nalgene, Waltham) containing $200 \mathrm{~mL}$ each of growth medium. Conditions for light and temperature were as described [15][17]. A. platensis NIES-39 was precultured in the boron-containing medium, harvested by filtration on nylon mesh as described [17], and washed on it with boron-depleted medium before inoculation into each growth medium. Anabaena sp. UTEX 2576 was cultured in the same way except that pre-cultured cells were collected and washed by centrifugation rather than by filtration.

\subsection{Quantitation of boron in the medium}

To draw standard curves, $900 \mu \mathrm{L}$ of $0.11 \mathrm{mM}$ $\mathrm{H}$-resorcinol in $0.65 \mathrm{M}$ ammonium acetate-acetic acid buffer ( $\mathrm{pH} 5.5)$ was mixed with $100 \mu \mathrm{L}$ of SOT medium containing various concentrations of boric acid. The mixtures were incubated at $25^{\circ} \mathrm{C}$ with constant shaking at $140 \mathrm{rpm}$. After $60 \mathrm{~h}$, optical density at the wavelength of $510 \mathrm{~nm}$ $\left(\mathrm{OD}_{510}\right)$ was determined with a spectrophotometer (Novaspec II, Pharmacia-LKB Biotechnology, Uppsala). Samples for quantitation were reacted with $\mathrm{H}$-resorcinol in the same way, and $\mathrm{OD}_{510}$ was determined. Means and the standard errors of means were determined from 4 samples.

\subsection{Determination of growth curves}

Cyanobacterial cells were cultured in four polycarbonate flasks for each set of culture conditions. The means and the standard deviations of the $\mathrm{OD}_{730}$ values and the trichome concentrations were determined over time. The numbers of trichomes in cultures were determined as described [18].

\subsection{Determination of the amounts of biomass, protein, chlorophyll $a$, and C-phycocyanin}

Samples were taken from cultures during the late logarithmic phase when the $\mathrm{OD}_{730}$ values of the cultures were approximately 1.0-1.2. Data were obtained from four independent cultures for each set of culture conditions, and the means and the standard deviations were determined.

To determine the amount of biomass, $10 \mathrm{~mL}$ of cultures were centrifuged at $4^{\circ} \mathrm{C}$ for $20 \mathrm{~min}$ at $3,000 \mathrm{~g}$, and the collected biomass was washed with $1 \mathrm{~mL}$ each of distilled water for 5 times. The washed biomass was dried at $85^{\circ} \mathrm{C}$ for $72 \mathrm{~h}$, cooled down, and weighed.

To determine the amount of proteins, cells in 1 $\mathrm{mL}$ of samples were collected by centrifugation as above and washed five times with $1 \mathrm{~mL}$ each of 25 $\mathrm{mM}$ Tris- $\mathrm{HCl}$ (pH 7.4), $138 \mathrm{mM} \mathrm{NaCl}, 2.68 \mathrm{mM}$ $\mathrm{KCl}$ (Tris-buffered saline). Then, they were disrupted by the Mini-Beadbeater-1 (Biospec Products, Bartlesville) using the $0.1 \mathrm{~mm}$ diameter glass beads. In the disruption, shaking the samples on the equipment for $30 \mathrm{sec}$ and cooling them on ice for $1 \mathrm{~min}$ were repeated 7 times. Protein concentrations in these samples were determined by Bradford's method [19] using Bio-Rad Protein Assay kit (Bio-Rad Laboratories, Hercules). As a standard for protein determination, bovine $\gamma$ globulin (Bio-Rad Laboratories) was used.

Concentrations of chlorophyll $a$ and Cphycocyanin were determined from the $\mathrm{OD}_{620}$ and $\mathrm{OD}_{678}$ values of the cultures as described [20]. 


\section{RESULTS}

\subsection{Quantitation of boron concentration in the growth medium}

In the experiments to follow, A. platensis was propagated in the medium with no added boric acid. However, it is known that considerable amount of boron is released from borosilicate glass into water when solutions are stored in glassware [13]. Therefore, the medium and all solutions were prepared in plasticware to avoid boron contamination from glassware. Even with this care, it was still possible that reagents used to prepare the medium were contaminated with boron. Therefore, boron concentration in the prepared growth medium was examined before performing the main experiments. To determine boron concentration, a spectrophotometric quantitation method with H-resorcinol was employed. This reagent forms a 1:1 complex with boron under weakly acidic conditions with an optimum at $\mathrm{pH}$ 5.5, and the complex can be determined spectrophotometrically at $510 \mathrm{~nm}$ [21]-[23].

Results of the boron quantitation in the borondepleted medium and in the usual medium (SOT medium) are shown in Fig. 1. As shown in the upper left panel, experiments to draw a calibration curve showed that the standard solutions containing as low as $0.2 \mu \mathrm{M}$ boric acid (12.4 ng $\mathrm{mL}^{-1}$ ) exhibited significant $\mathrm{OD}_{510}$ values, indicating that detection limit for boric acid with this method was lower than $0.2 \mu \mathrm{M}$. In contrast,
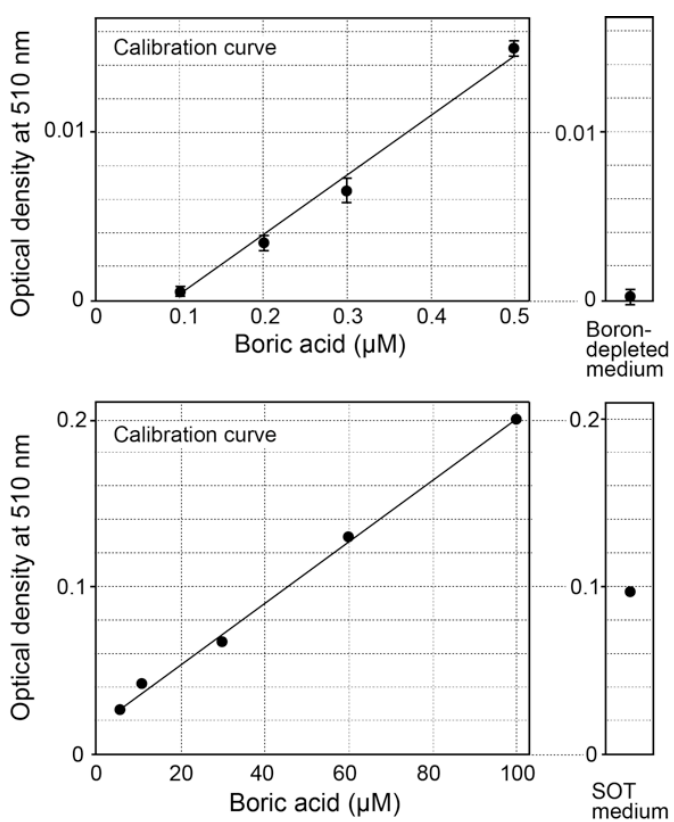

Fig.1 Quantitation of boron concentrations
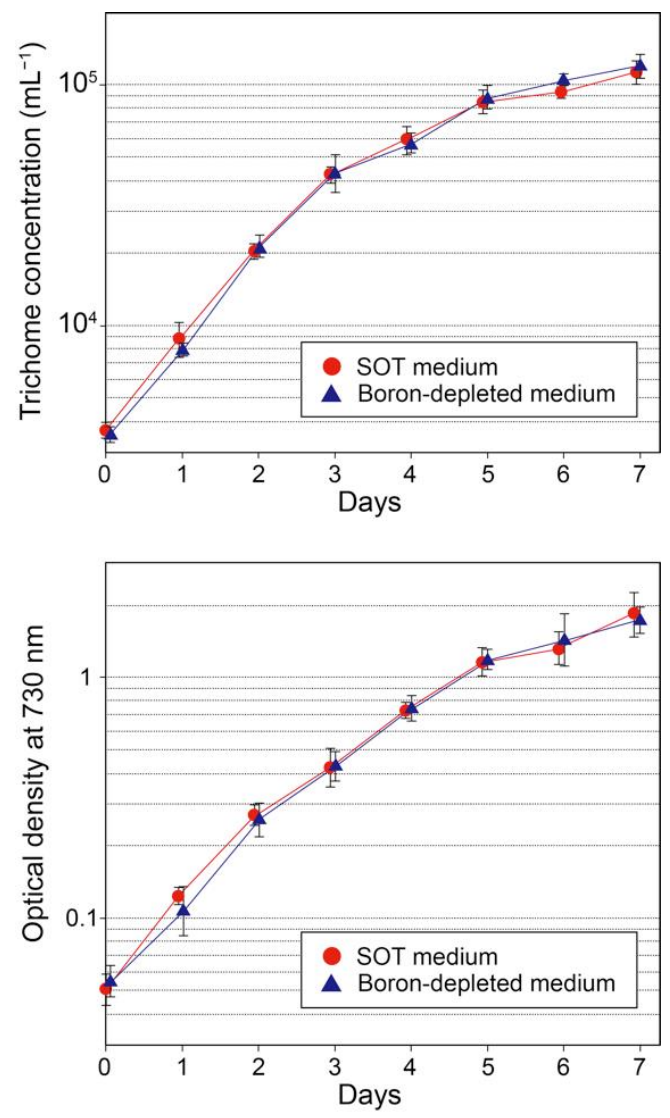

Fig. 2 Growth of A. platensis in relation to boron

samples prepared with the boron-depleted medium exhibited hardly detectable absorption at $510 \mathrm{~nm}$ (Fig. 1, upper right panel). Therefore it was clear that boric acid concentration in this medium was far lower than $0.2 \mu \mathrm{M}$. In this respect, it has been reported that, when water was stored in glass bottles, concentration of boron released from glass into water reached $5.2 \mu \mathrm{M}$ [13]. Therefore, the boron level in the boron-depleted medium $(<0.2$ $\mu \mathrm{M})$ was far lower than the residual level of boron in water stored in glass bottles. Concentration of boric acid in the SOT medium used in this study was also examined and determined to be approximately $45 \mu \mathrm{M}$ (Fig. 1, lower panels).

3.2 Effect of boron depletion on the growth of $A$. platensis NIES-39 and Anabaena sp. UTEX 2576

To examine the effect of boron on the growth of A. platensis, A. platensis NIES-39 was cultured in the usual SOT medium and in the borondepleted medium prepared as above. Trichome concentrations and the $\mathrm{OD}_{730}$ values were determined over time, and growth curves were drawn. As shown in Fig. 2, there were no significant differences between the growth curves 


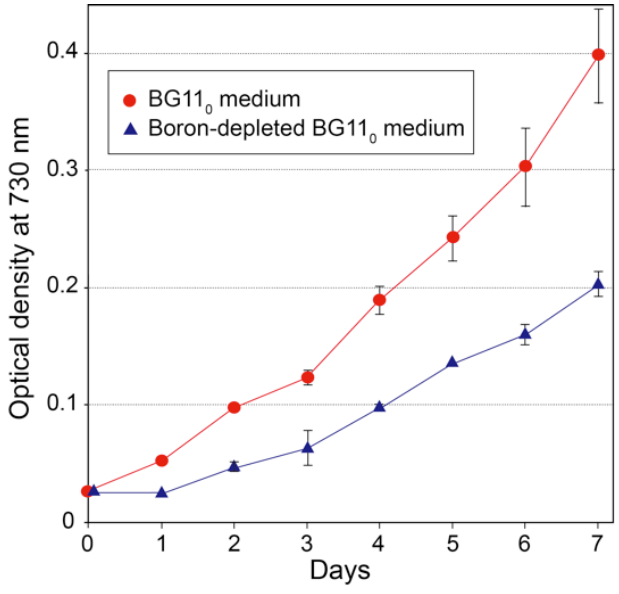

Fig. 3 Growth of Anabaena sp. in relation to boron

determined in the presence and absence of boron, indicating that boron depletion had no detectable effect on the growth of A. platensis NIES-39.

As a control experiment, a heterocystous cyanobacterium Anabaena sp. UTEX 2576 was cultured in the boron-containing medium $\left(\mathrm{BG} 11_{0}\right.$ medium) and in the boron-depleted $\mathrm{BG} 11_{0}$ medium As shown in Fig. 3, growth of this cyanobacterium was suppressed in the boron-depleted medium. Statistical analysis by the Student's $t$-test indicated significant differences $(P<0.05)$ between the cultures in the BG11 $1_{0}$ medium and in the borondepleted $\mathrm{BG} 11_{0}$ medium at and after $24 \mathrm{~h}$. This control experiment confirmed that boron had been effectively depleted in the boron-depleted media prepared in this study. It is worth noting that the culture of Anabaena sp. UTEX 2576 grown in the boron-depleted medium exhibited a yellowish color, similar to other heterocystous cyanobacteira grown under boron-depleted conditions [6][7]. In contrast, the culture of $A$. platensis NIES-39 had the usual blue-green color even when it was propagated in the boron-depleted medium.

The experiments in Fig. 2 and Fig. 3 clearly indicated that boron depletion had no detectable effect on the growth of A. platensis whereas it deteriorated the growth of Anabaena sp. that requires boron for the $\mathrm{N}_{2}$-fixation-dependent growth.

\subsection{Effect of boron depletion on the biomass and the protein, chlorophyll $a$, and C- phycocyanin contents}

A. platensis trichomes propagated in the borondepleted medium were visually indistinguishable from those propagated in the usual SOT medium, when their morphologies were observed under a dissecting microscope. To examine whether there were any differences in their cellular physiology, some biochemical properties were determined next.
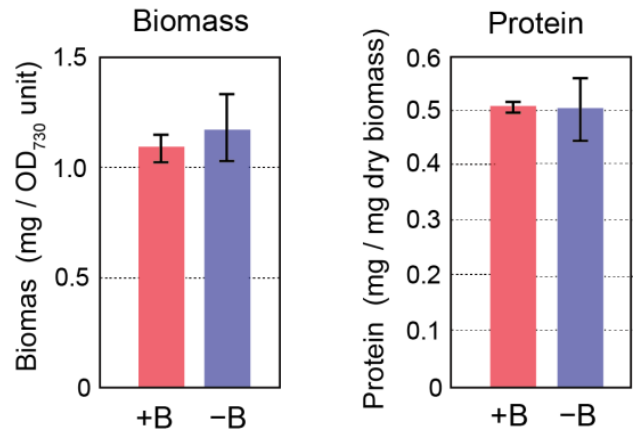

Fig.4 Biomass and the protein content

Fig. 4 shows the amount of biomass and the protein content of A. platensis NIES-39 propagated in the presence and absence of boron. There were no significant differences between them as analyzed by the Student's $t$-test with significance level set at 0.05 . The protein content of $A$. platensis NIES-39 was approximately $50 \%(\mathrm{w} / \mathrm{w})$ both in the presence and absence of boron (Fig. 4, right panel). These values were comparable to the values $(50-55 \%)$ that had been determined with another A. platensis strain [24].

The Chlorophyll $a$ and C-phycocyanin contents were also determined and compared (Fig. 5). Analysis by the Student's $t$-test with significance level set at 0.05 indicated that there were again no significant differences in their contents between the cells cultured in the presence and absence of boron.

\section{DISCUSSION}

The boron requirement by A. platensis has not been carefully examined. Whereas there has been a report that boron can be omitted from the growth medium to culture A. platensis PCC 8005 , experiments in that study were performed using a glass fermenter [25]. Since water becomes contaminated with boron when stored in glass containers and that the boron release from glass is accelerated under alkaline conditions [13], it was
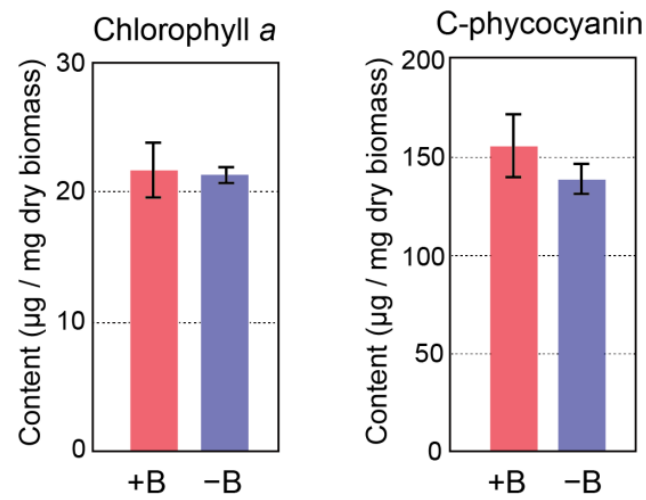

Fig. 5 Chlorophyl $a$ and C-phycocyanin contents 
possible that the boron released from the glass vessel into the alkaline-leaned medium for $A$. platensis was sufficient to support the normal growth of A. platensis in that study. In contrast, our study was carefully performed using plasticware to avoid boron contamination. Our experimental results clearly demonstrated that even a residual amount of boron is not necessary for the growth of A. platensis (Fig. 2). In addition, there were no detectable differences in the biochemical properties of the cells grown in the presence and absence of boron (Fig. 4 and Fig. 5).

It has been reported that the $\mathrm{C}$-phycocyanin content of many Arthrospira strains significantly increased when they were cultured in a medium that was not supplemented with microelements (Fe $\mathrm{B}, \mathrm{Mn}, \mathrm{Zn}, \mathrm{Cu}, \mathrm{Mo}$ ) [26]. This effect was most likely caused by the limitation of the element(s) other than boron, since our study demonstrated that boron depletion had no significant effect on the C-phycocyanin content.

A. platensis has been used for commercial propagation. In addition to commercial usage, it has also been used as a small-scale crop providing nutritional supplements for the communities where the staple diet is poor or inadequate [27][28]. The results of our study suggest that $A$. platensis can be an excellent crop that can be grown without boron supplementation even in the region where boron deficiency in the soil deteriorates the healthy growth of many common crops.

In the studies of Arthrospira, synthetic growth media used in biological experiments have been supplemented with boric acid [3][14]. However, boric acid gradually forms an insoluble salt manganese borate [10], since the growth media contain manganese ion. Formation of the precipitate of manganese borate may gradually deplete manganese ions in the media during prolonged storage. This may affect the results of sensitive physiological experiments, e.g., metabolome analysis, because manganese is an essential cofactor of many metabolic enzymes [11][12]. Since our study showed that boron is not necessary for the growth of $A$. platensis, the use of boron-free media is recommended in sensitive physiological studies of $A$. platensis.

\section{CONCLUSION}

Boron depletion had no detectable effect on the growth and biochemical properties of A. platensis. This result indicates that boron supply is not necessary for the propagation of $A$. platensis even in the regions where boron deficiency in the soil deteriorates the growth of common crops. Since boron is not necessary for the growth of $A$. platensis, the use of boron-free media is recommended when performing sensitive physiological experiments, to avoid the precipitation of manganese borate that may reduce effective concentration of manganese ions in the media.

\section{ACKNOWLEDGEMENTS}

This work was supported by the Grant-in-Aid for Challenging Exploratory Research from the Japan Society for the Promotion of Science [JSPS KAKENHI Grant Number 26660063] and the Management Expenses Grants for National University Corporations from the Ministry of Education, Culture, Sports, Science and Technology of Japan.

\section{REFERENCES}

[1] Belay A, "Biology and industrial production of Arthrospira (Spirulina)", Handbook of Microalgal Culture: Applied Phycology and Biotechnology, 2nd ed. Richmond $\mathrm{A}$ and $\mathrm{Hu} \mathrm{Q}$, Eds. West Sussex: Wiley-Blackwell, 2013, pp. 339-358

[2] Grewe CB and Pulz O, "The Biotechnology of Cyanobacteria", Ecology of Cyanobacteria II: Their Diversity in Space and Time. Whitton BA, Ed. Dordrecht: Springer, 2012, pp. 707739.

[3] Zarrouk C, Contribution à l'étude d'une cyanophycée. Influence de divers facteurs et chimiques sur la croissance et la photosynthèse de Spirulina maxima Geilter. PhD thesis, Université de Paris. Paris, 1966.

[4] O’Neill MA, Ishii T, Albersheim P and Darvill AG, Rhamnogalacturonan II: Structure and function of a borate cross-linked cell wall pactic polysaccharide. Annu. Rev. Plant. Biol. Vol. 55, 2004, pp. 109-139.

[5] Shorrocks VM, The occurrence and correction of boron deficiency. Plant and Soil, Vol. 193, 1997, pp. 121-148.

[6] Mateo P, Bonilla I, Fernández-Valiente E and Sanchez-Maeso, Essentiality of boron for dinitrogen fixation in Anabaena sp. PCC 7119. Plant Physiol. Vol. 81, 1986, pp. 430-433.

[7] Bonilla I, Garcia-González M and Mateo P, Boron requirement in cyanobacteria: Its possible role in the early evolution of photosynthetic organisms. Plant Physiol. Vol. 94, 1990, pp. 1554-1560.

[8] Garcia-Gonzalez M, Mateo P and Bonilla I, Boron requirement for envelope structure and function of Anabaena PCC 7119 heterocysts. J. Exp. Bot. Vol. 42, 1991, pp. 925-929.

[9] Martinez F, Mateo P, Bonilla I, FernandezValiente E and Garate A, Growth of Anacystis 
nidulans in relation to boron supply. Israel $\mathrm{J}$. Botany, Vol. 35, 1986, pp. 17-21.

[10] The Merck Index: An Encyclopedia of Chemicals, Drugs and Biologicals, 13th ed. O'Neil MJ, Smith A, Heckelman PE and Budavari S., Eds. Whitehouse Station: Merck and Co. Ltd., 2001.

[11] Yachandra VK, Sauer K and Klein MP, Manganese cluster in photosynthesis: Where plants oxidize water to dioxygen. Chem. Rev. Vol. 96, 1996, pp. 2927-2950.

[12] Penner-Hahn JE, "Manganese proteins with mono- \& dinuclear sites", Encyclopedia of Inorganic Chemistry, 2nd ed, Vol. V, King RB Ed. West Sussex: Wiley, 2005, pp. 2922-2932.

[13] Green GH, Blincoe C and Weeth HJ, Boron contamination from borosilicate glass. J. Agric. Food Chem. Vol. 24, 1976, pp. 1245-1246.

[14] Ogawa T and Terui G, Studies on the growth of Spirulina platensis. (I) On the pure culture of Spirulina platensis. J. Ferment. Technol. Vol. 48, 1970, pp. 361-367.

[15]Shiraishi $\mathrm{H}$ and Tabuse $\mathrm{Y}$, The AplI restriction-modification system in an edible cyanobacterium, Arthrospira (Spirulina) platensis NIES-39, recognizes the nucleotide sequence 5'-CTGCAG-3'. Biosci. Biotechnol. Biochem. Vol. 77, 2013, pp. 782-788.

[16]Rippka R, Deruelles J, Waterbury JB, Herdman $M$ and Stanier RY, Generic assignments, strain histories and properties of pure cultures of cyanobacteria. J. Gen. Microbiol., Vol. 111, 1979, pp. 1-61.

[17] Shiraishi H, Association of heterotrophic bacteria with aggregated Arthrospira platensis exopolysaccharides: Implications in the induction of axenic cultures. Biosci. Biotechnol Biochem. Vol. 79, 2015, pp. 331-341.

[18] Shiraishi, H, Cryopreservation of the edible alkalophilic cyanobacterium Arthrospira platenisis. Biosci. Biotechnol. Biochem. Vol. 80, 2016, pp. 2051-2057.

[19] Bradford MM, Rapid and sensitive method for the quantitation of microgram quantities of protein utilizing the principle of protein-dye binding. Anal. Biochem. Vol. 72, 1976, pp. 248-254.

[20] Arnon DI, McSwain BD, Tsujimoto HY and Wada K, Photochemical activity and components of membrane preparations from blue-green algae. I. Coexistance of two photosystems in relation to chlorophyll $a$ and removal of phycocyanin. Biochim. Biophys. Acta, Vol. 357, 1974, pp. 231-245.

[21] Capelle K, Microdosage colorimétrique du bore en milieu aqueux, au moyen de réactifs a groupement azoïque ou imine dérivés des acides H et K. Anal. Chim. Acta, Vol. 24, 1961, pp. 555-572.

[22] Tôei K, Motomizu S, Oshima M and Onoda M, Spectrophotometric determination of boron by flow injection analysis. Bunseki Kagaku, Vol. 35, 1986, pp. 344-348.

[23] Motomizu S, Oshima M and Jun Z, Anionexchange chromatographic behaviour of the complex of boron with H-resorcinol: sensitive determination of boric acid. Analyst, Vol. 115, 1990, pp. 389-392.

[24] Anusuya Devi M, Subbulakshmi G, Madhavi Devi K and Venkataraman LV, Studies on the proteins of mass-cultivated, blue-green alga (Spirulina platensis). J. Agric. Food Chem. Vol. 29, 1981, pp. 522-525.

[25] Cogne G, Lehmann B, Dussap C-G and Gros $\mathrm{J}-\mathrm{B}$, Uptake of macrominerals and trace elements by the cyanobacterium Spirulina platensis (Arhtrospira platensis PCC 8005) under photoautotrophic conditions: Culture medium optimization. Biotech. Bioengineer. Vol. 81, 2003, pp. 588-593.

[26] Tarko T, Duda-Chodak A and Kobus M, Influence of growth medium composition on synthesis of bioactive compounds and antioxidant properties of selected strains of Arthrospira Cyanobacteria. Czech J. Food Sci. Vol. 30, 2012, pp. 258-267.

[27] Jeeji Bai N, Seshadri CV, Small scale culture of Spirulina (Arthrospira) as food supplement for rural households-Technology development and transfer. Algol. Stud. Vol 50-53, 1988, pp. 565-572.

[28] Habib MAB, Parvin M, Huntington TC and Hasan MR, A review on culture, production and use of spirulina as food for humans and feeds for domestic animals and fish (FAO Fisheries and Aquacultre Circular No. 1034). Rome: Food and Agriculture Organization of the United Nations, 2008.

Copyright (C) Int. J. of GEOMATE. All rights reserved, including the making of copies unless permission is obtained from the copyright proprietors. 